\title{
Development of synthetic light-chain antibodies as novel and potent HIV fusion inhibitors
}

\author{
Catarina Cunha-Santos ${ }^{a}$, Tiago N. Figueirab ${ }^{b}$ Pedro Borrego ${ }^{a, c}$, \\ Soraia S. Oliveira ${ }^{a}$, Cheila Rocha ${ }^{a, c}$, Andreia Couto ${ }^{a}$, Cátia Cantante ${ }^{a}$, \\ Quirina Santos-Costa ${ }^{a}$, José M. Azevedo-Pereira ${ }^{a}$, \\ Carlos M.G.A. Fontes ${ }^{\mathrm{d}}$, Nuno Taveira ${ }^{\mathrm{a}, \mathrm{c}}$, Frederico Aires-Da-Silva ${ }^{\mathrm{d}, \mathrm{e}}$, \\ Miguel A.R.B. Castanho ${ }^{b}$, Ana Salomé Veiga ${ }^{b}$ and Joao Goncalves ${ }^{a}$
}

\begin{abstract}
Objective: To develop a novel and potent fusion inhibitor of HIV infection based on a rational strategy for synthetic antibody library construction.

Design: The reduced molecular weight of single-domain antibodies (sdAbs) allows targeting of cryptic epitopes, the most conserved and critical ones in the context of HIV entry. Heavy-chain sdAbs from camelids are particularly suited for this type of epitope recognition because of the presence of long and flexible antigen-binding regions [complementary-determining regions (CDRs)].

Methods: We translated camelid CDR features to a rabbit light-chain variable domain $(\mathrm{VL})$ and constructed a library of minimal antibody fragments with elongated CDRs. Additionally to elongation, CDRs' variability was restricted to binding favorable amino acids to potentiate the selection of high-affinity sdAbs. The synthetic library was screened against a conserved, hidden, and crucial-to-fusion sequence on the heptad-repeat 1 (HR1) region of the HIV-1 envelope glycoprotein.

Results: Two anti-HR1 VLs, named F63 and D104, strongly inhibited laboratoryadapted HIV-1 infectivity. F63 also inhibited infectivity of HIV-1 and HIV-2 primary isolates similarly to the Food and Drug Administration-approved fusion inhibitor T-20 and HIV-1 strains resistant to T-20. Moreover, epitope mapping of F63 revealed a novel target sequence within the highly conserved hydrophobic pocket of HR1. F63 was also capable of interacting with viral and cell lipid membrane models, a property previously associated with T-20's inhibitory mechanism.

Conclusion: In summary, to our best knowledge, we developed the first potent and broad VL sdAb fusion inhibitor of HIV infection. Our study also gives insights into engineering strategies that could be explored to enhance the development of antiviral drugs.

Copyright (c) 2016 Wolters Kluwer Health, Inc. All rights reserved.
\end{abstract}

AIDS 2016, 30:000-000

Keywords: HIV fusion inhibitor, HR1, single-domain antibody, synthetic library, variable light-chain

\footnotetext{
${ }^{a}$ Research Institute for Medicines (iMed.ULisboa), Faculty of Pharmacy, ${ }^{b}$ Instituto de Medicina Molecular, School of Medicine, Universidade de Lisboa, Lisboa, 'ISCSEM-Centro de Investigação Interdisciplinar Egas Moniz, Instituto Superior de Ciências da

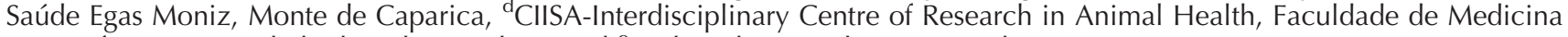
Veterinária, Universidade de Lisboa, Lisboa, and ${ }^{\mathrm{e}}$ Technophage, Lisboa, Portugal.

Correspondence to Joao Goncalves, iMed-Research Institute for Medicines, Avenida Prof. Gama Pinto, 1649-003 Lisbon, Portugal. Tel: +351 217946 400; e-mail: jgoncalv@ff.ulisboa.pt Received: 24 November 2015; revised: 18 March 2016; accepted: 31 March 2016.
} 


\section{Introduction}

Significant advances in antiretroviral therapy have occurred as the approval of the first fusion inhibitor, $\mathrm{T}-20$ [1]. T-20 peptide derives from the C-terminal region (HR2) of gp41 fusion protein from $\mathrm{HIV}_{643-678(\mathrm{LAI})}[2,3]$. By competitively binding the N-terminal region (HR1), T-20 impairs HR 1-HR 2 interaction [2] and consequently the formation of the six-helix bundle $(6 \mathrm{HB})$ structure, responsible for HIV fusion (reviewed in Wilen et al. [4]). Despite the well characterized antiviral potency of T-20, clinical resistance has been reported in HIV-1-infected patients [5]. Additionally, T-20 is described as antigenic [6], highly expensive, protease-susceptible (no oral administration), and 'pharmacokinetically limited', among other limitations [7]. Overall, development of novel HIV fusion inhibitors with improved biophysical and pharmacokinetic properties is required.

Antibody fragments emerged to overcome issues associated with high-molecular weight of native antibody structure ( $\operatorname{IgG})$, mainly the targeting of cryptic epitopes and the penetration into densely packed tissues. Singledomain antibody ( $\mathrm{sdAb}$ ) is currently the smallest functional antibody fragment, only constituted by the antibody heavy-chain or light-chain variable domains (VH or VL) [8]. Additionally to the reduced size, complementarydetermining regions (CDRs; antigen-binding regions) of dAbs can be easily engineered to develop specific and highaffinity binders. sdAbs also present excellent biophysical properties, such as high stability, solubility, and low toxicity [9]. Despite these beneficial features, only the therapeutic potential of $\mathrm{VH}$ domains have been intensively explored [10]. Nevertheless, several reports have demonstrated that VL domains present excellent biophysical properties, such as high expression yield, resistance to aggregation and proteases, stability, and high reversibility of thermal unfolding, in some cases better than VHs [11-14]. Moreover, the stability of VL domains was further evidenced by the proved functionality of these $\mathrm{dAb}$ in the absence of disulfide bonds $[15,16]$ or in the reducing cellular environment $[17,18]$.

Here, we engineered a VL sdAb with elongated CDRs that broadly and potently inhibits HIV-1 infection by targeting a well conserved and crucial-to-fusion sequence on HR1. Despite the clinical resistance to HR1-targeting $\mathrm{T}-20$, this region contains highly conserved residues among HIV-1 subtypes and isolates [19], representing a major target to HIV infection impairment. Anti-HR1 VLs were selected by phage display technology from a restricted combinatorial library. Epitope mapping of the two most potent antiviral VLs - selected against an HIV-1 laboratory-adapted strain - showed that these inhibitors target a highly conserved and critic region within HR 1 . One VL (F63) showed high potency to inhibit HIV-1 and HIV-2 primary isolates with comparable T-20 activity. For last, we demonstrated that F63 also interacts with lipid membranes, a key ability of potent HIV entry inhibitors $[20,21]$ that correlates with their mechanism of action.

\section{Methods}

\section{Inhibition assays}

HIV-1 laboratory-adapted strain NL4-3 (HIV-1 $\left.{ }_{\mathrm{NL4}-3}\right)$ production was performed as described [22] and 50\% tissue culture infectious dose determined as Borrego et al. [6]. HIV-2 and HIV-1 primary isolates from subtypes $\mathrm{J}$ and $\mathrm{H}$ were obtained from Borrego et al. [6]. HIV-1 variant NL43 D36G (parental) and HIV-1 variants resistant to T-20 NL4-3 (D36G) V38A/N42D and V38A/N42T (NIH AIDS Reagent Program) were propagated accordingly to Borrego et al. [6]. HIV-1 primary isolates from subtypes B and $\mathrm{C}$ were obtained from Calado et al. [23].

For all the inhibition assays, viruses or HeLa243env/ $\mathrm{HeLa} 273 \Delta$ env cells were incubated with titrated amounts of the inhibitors during $1 \mathrm{~h}$ at $37^{\circ} \mathrm{C}$ prior to infection and HIV infectivity was measured $48 \mathrm{~h}$ postinfection. In Jurkat E6-1 (NIH AIDS Reagent Program) inhibition assay, HIV p24 ${ }^{\mathrm{CA}}$ concentrations were measured by ELISA (NCI, Frederick, Maryland, USA) according to manufacturer's instructions. In inhibition assays with TZM-bl cells (NIH AIDS Reagent Program) [6], luciferase or ' $\beta$-galactosidase' expression was quantified with the One-Glow luciferase assay substrate reagent (Promega, USA) according to manufacturer's instructions or as described in Da Silva et al. [24], respectively. Cell-cell fusion assay was adapted from Schwartz et al. [25]. HeLa243env or HeLa273senv cells [25] were cocultured at a $1: 1$ ration with multinuclear activation of a galactosidase indicator (MAGI) cells (NIH AIDS Reagent Program) in the presence of inhibitors. After $48 \mathrm{~h}$, ' $\beta$-galactosidase' expression was quantified as described [24].

Peripheral blood mononuclear cells' isolation, maintenance, and inhibition assays were performed as previously described [23] with the following exception: at 7 days postinfection, HIV p $24{ }^{\mathrm{CA}}$ concentrations were measured by ELISA. The $50 \%$ inhibitory concentration $\left(\mathrm{IC}_{50}\right)$ estimation and statistical analysis were performed as described $[6,26]$.

The remaining experimental procedures are provided in the Supplementary Methods (http://links.lww.com/ QAD/A912).

\section{Results}

Selection of anti-HIV VLs with elongated complementary-determining region 1 and complementary-determining region 3

In contrast to regular binding regions, long and flexible CDR3 of camelid heavy-chain antibodies [27] can 
successfully target hidden and nonstandard (immuneevasion) epitopes $[9,28]$. We proposed to translate these CDR features to a noncamelid scaffold - a rabbit $\mathrm{\kappa}$ VL domain [29] derived from a previously selected singlechain variable fragment [30]. In addition to the stability already attributed to VL sdAbs [11-14], their solubility seems less affected by sequence variation in CDRs than $\mathrm{VH}$ domains [14]. We chose a nonhuman domain because of the extensive CDR3 length heterogeneity naturally present in the $\kappa$ light chains of rabbit antibodies, in contrast with human ones $[31,32]$. Furthermore, this particular VL domain was shown to be highly stable and soluble in the absence of its counterpart VH domain [29]. The naturally longer and most exposed CDR of parental VL (CDR3; Fig. S1A, http://links.lww.com/QAD/ A912), hereafter named $\mathrm{VL}_{\text {parental, }}$, was grafted with a series of long CDRs containing a well characterized paratope for hen egg-white lysozyme [33] flanked by sequences of serines/glycines. These small amino acids major contributors to conformational flexibility of antibody CDRs [34,35] - were added to elongate the original CDR 3 of 11 amino acids to 22,26 , or 30 amino acids (Fig. S1B, http://links.lww.com/QAD/A912). Evaluation of $\mathrm{VL}_{\text {parental }}$ functionality in the presence of an elongated CDR3 is presented in Supplementary Information.

After validation of $\mathrm{VL}_{\text {parental }}$ functionality in the presence of an elongated CDR3, we used it as a scaffold for synthetic library construction. This library was designed to select anti-HIV minimal antibody fragments with high-affinity toward a cryptic HR 1 region. We chose to elongate both CDR 1 and CDR 3 to increase theoretically the affinity of the selected $\mathrm{dAbs}$ and at the same time avoid unspecific binding from the original CDR1. For the CDRs library construction, we used the previously validated strategy for hen egg-white lysozyme paratope grafting (Fig. 1a), restricted randomization of the central 12 amino acids with a degenerate codon (DVN) that only encodes for 12 of the canonical 20 amino acids. As most encoded amino acids by DVN codon were described as abundant in natural CDRs and antigenic contacts [36], we expected to improve the selection of high-affinity sdAbs. Moreover, these amino acids seem to be sufficient to generate high-affinity and specific minimalist synthetic binders $[35,36]$. A library of $\sim 8.0 \times 10^{9}$ clones was generated, cloned, and selected by phage display against a crucial-to-fusion, difficult-to-access, and well conserved sequence on HR1 (HR1 $\left.1_{546-581(\mathrm{HXB} 2)}\right)$, named N36 [19] (Fig. S2, http://links.lww.com/QAD/A912). Apart from the cryptic nature of the entire HR1 region, N36 comprises residues of a particularly deep cavity, named hydrophobic pocket, described as highly conserved and a hot spot for neutralization of HIV-1 infection [19,37]. Despite the therapeutic interest, this pocket is particularly difficult to target in an infection context because of its extreme concave conformation. As shown in Fig. 1b, we isolated five VLs with strong binding to HR1 from the
329 clones screened by ELISA. A further characterization of HR1 binding showed a dose-dependent binding for all five selected VLs (Fig. 1c and Table S2, http:// links.lww.com/QAD/A912). A competitive ELISA demonstrated that the five VLs showed a decreased binding to immobilized HR 1 as the soluble HR 1 amount increased, confirming VLs specificity of recognition (Fig. 1c).

To assess the antiviral activity of selected VLs, we performed a second screening ('functional screening') against HIV-1 $1_{\mathrm{NL} 4-3}$ - encoding the G547D mutation responsible for less susceptibility to T-20 fusion inhibitor [38]. From the five anti-HR1 VLs, F63 and D104 inhibited HIV-1 $1_{\mathrm{NL} 4-3}$ infectivity by $\sim 90 \%$ (Fig. 1d) and were selected for further characterization of antiviral activity. Except for CDR1 of VL D103 that was not randomized, DNA sequencing analysis confirmed that all CDR 1 and CDR 3 sequences of the five anti-HR 1 VLs were unique (Fig. 1e). F63 and D104 VLs were expressed and purified in high yield and used for further functional characterization (Fig. S3, http://links.lww.com/QAD/ A912).

\section{Epitope mapping of antiviral VLs}

Epitopes of F63 and D104 were mapped by ELISA, using a set of 10 overlapping synthetic peptides covering the template HR1 (Fig. 2a), and compared with the T-20 binding region. Both VLs exhibited similar target sequences within the central region of N36 with short overlap at the C-terminus of T-20 binding region (Fig. 2b and c). F63 showed the strongest binding to peptide $5\left(\mathrm{NH}_{2}\right.$-EAQQHMLQLTVWGIK-COOH), suggesting that it might contain its epitope (Fig. 2b). D104 showed similar binding to peptides 5 and 6 (Fig. 2b), indicating that its epitope is located within the overlapping sequence of 11 amino acids $\mathrm{NH}_{2}-\mathrm{HMLQLTVWGIK-COOH}$ (Fig. 2a).

\section{Antiviral activity of VLs}

The antiviral activity of VLs was first compared with T-20 peptide against the HIV-1 $1_{\text {NL4-3. }}$. T-20 also binds HR1 impairing the virus-cell fusion driven by HR1-HR2 interaction, an inhibition mechanism we reasoned to be similar to selected anti-HIV VLs. As shown in Fig. 3a, both F63 and D104 strongly inhibited HIV-1 ${ }_{\text {NL4-3 }}$ infection in TZM-bl cells ( $\mathrm{IC}_{50}$ was $0.5 \pm 0.2 \mathrm{nmol} / 1$ for $\mathrm{F} 63$ and $9.7 \pm 5.4 \mathrm{nmol} / 1$ for D104). Remarkably, F63 was more active against $\mathrm{HIV}-1_{\mathrm{NL} 4-3}$ than D104 and T-20 $\left(\mathrm{IC}_{50}\right.$ was $0.5 \pm 0.2 \mathrm{nmol} / 1$ for F63 vs. $9.7 \pm 5.4 \mathrm{nmol} / 1$ for D104 and $3.1 \pm 1.9 \mathrm{nmol} / 1$ for $\mathrm{T}-20)$. As expected, the $\mathrm{VL}_{\text {parental }} \mathrm{did}$ not inhibit HIV-1 ${ }_{\mathrm{NL} 4-3}$. F63 and D104 also strongly inhibited HIV-1 infection similarly to T-20 in Jurkat cells $\left(\mathrm{IC}_{50}\right.$ was $0.1 \pm 0.01 \mathrm{nmol} / 1$ for $\mathrm{F} 63,0.6 \pm 0.1 \mathrm{nmol} / 1$ for D104, and $0.1 \pm 0.01 \mathrm{nmol} / 1$ for T-20; Fig. 3b). No cytotoxicity was observed when either TZM-bl or Jurkat cells were incubated with the highest concentration of the VLs (Fig. S4, http://links.lww.com/QAD/A912). 


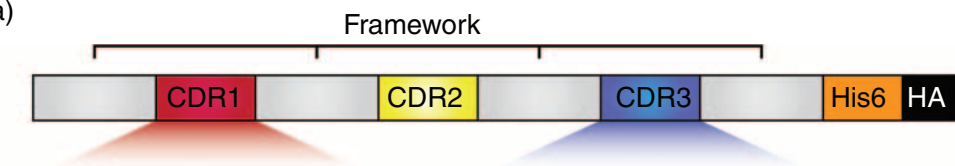

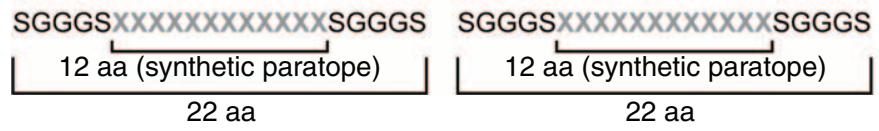

(c)
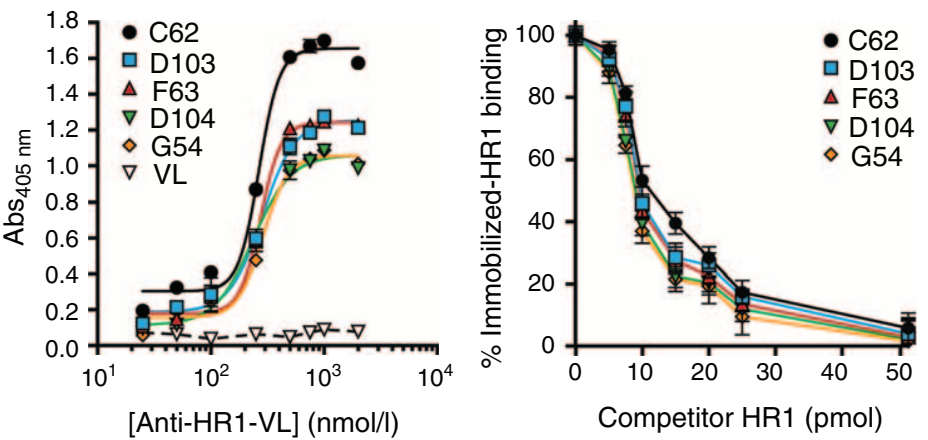

(d)

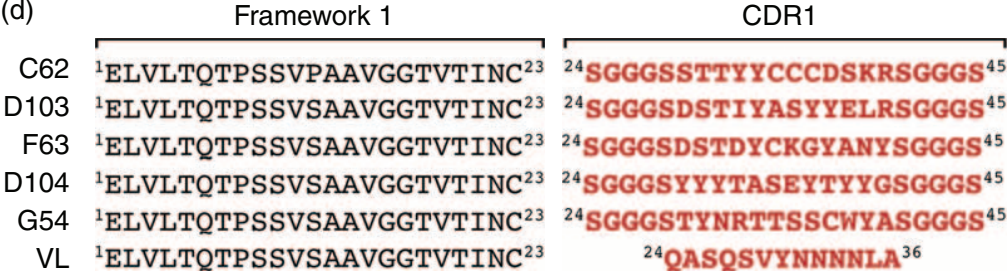
${ }^{24}$ QASQSVYNNNNLA ${ }^{36}$

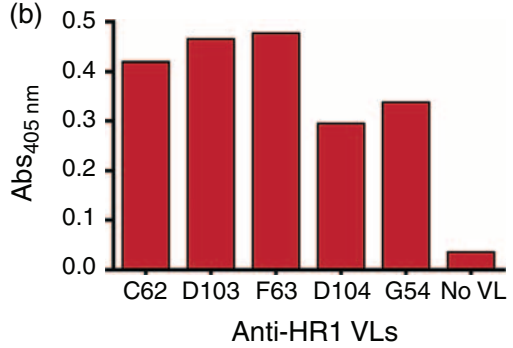

(d)

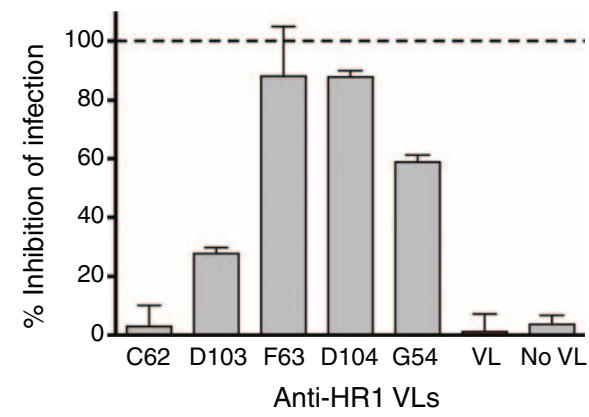

Framework 2

CDR2

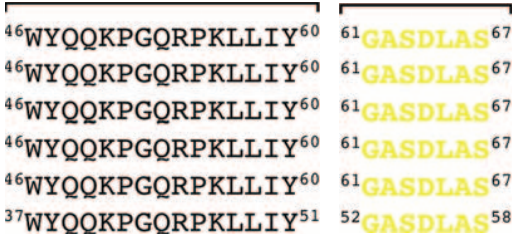

${ }^{37}$ WYQOKPGORPKLLIY ${ }^{51} \quad 52$ CARP 38

CDR3



Fig. 1. Selection of anti-HIV VLs from the constructed synthetic library. (a) Schematic representation of VLs synthetic library. CDR1 and CDR3 were randomized in the central 12 amino acids represented by the $\mathrm{X}$ letter in grey. Serines/glycines sequence was added to the flanks to provide flexibility. The hexahistidine tail (His6) was used for further purification of the VLs and hemagglutinin peptide sequence tag for detection. (b) Anti-HR1 VLs selection. The anti-HR1 VLs were expressed in bacteria and cell extracts incubated with N36 region of HR1 or BSA in ELISA plates. No VL represents no VL expression. The five phage-selected VLs out of 329 that presented highest binding values to HR1 are represented. Data are displayed as Abs measurement at $405 \mathrm{~nm}$. To facilitate data representation, HR1 binding was calculated according to the following formula: Abs HR1-coated well $_{\text {Abs }}$ BSA-coated well. (c) HR1-binding analysis. Increasing concentrations of the purified five selected VLs and control $\mathrm{LL}_{\text {parental }}(\mathrm{VL})$ were incubated with HR1 or BSA-coated wells (left). Data are displayed as Abs measurement at $405 \mathrm{~nm}$. To facilitate data representation, HR1 binding was calculated according to

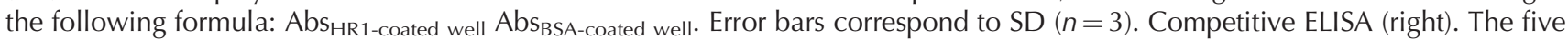
selected $V L s$ and $V L_{\text {parental }}(\mathrm{VL})$ were preincubated with increasing quantities of soluble $\mathrm{N} 36$ region of $\mathrm{HR} 1$ at $37^{\circ} \mathrm{C}$. After $1 \mathrm{~h}$, this mixture was incubated with N36-coated wells (immobilized-HR1). Data are displayed as percentage of immobilized-HR1 binding (no competitor/immobilized-HR1 $=100 \%$ ) according to formula: [( $\mathrm{Abs}_{\text {competitor/immobilized-HR1 }}-\mathrm{Abs}_{\text {competitor/immobilized }}$ BSA $)$ / $\left.\left(\mathrm{Abs}_{\text {nocompetitor/immobilized-HR1 }}-\mathrm{Abs}_{\text {nocompetitorimmobilized BSA }}\right)\right] \times 100$. Error bars correspond to SD $(n=3)$. (d) Selection of antiviral

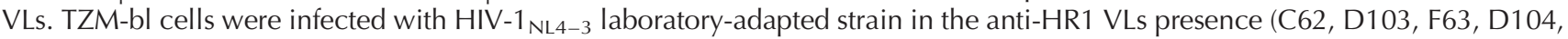
and G54). TZM-bl cell line expresses ' $\beta$-galactosidase' gene under control of HIV-1 promoter (long terminal repeat) - activated in the presence of HIV transactivator of transcription (Tat) protein (infection). $\mathrm{VL}_{\text {parental }}(\mathrm{VL})$ represents the negative control. No $\mathrm{VL}$ represents no VL expression. Data are displayed as percentage of infectivity inhibition (virus/no inhibitors $=0 \%$ inhibition; no virus/ no inhibitors $=$ background $)$ according to the formula: $\left[\left(\mathrm{Abs}_{\text {virus/inhibitors }}-\mathrm{Abs}_{\text {background }}\right) /\left(\mathrm{Abs}_{\text {virus/noinhibitors }}-\mathrm{Abs}_{\text {background }}\right)\right] \times 100$. Error bars correspond to SD $(n=2)$. (e) Amino acid sequences of the five anti-HR1 VLs: C62, D103, F63, D104, G54, and control $\mathrm{VL}_{\text {parental }}(\mathrm{VL})$. CDR1 is highlighted in red, CDR2 in yellow, and CDR3 in blue. VLs backbone composed of four frameworks is represented in grey. CDR, complementary-determining region; SD, standard deviation. 
(a)

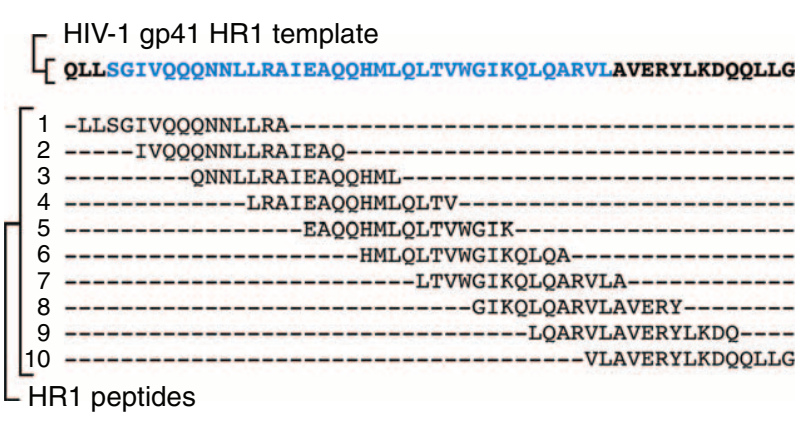

(b)
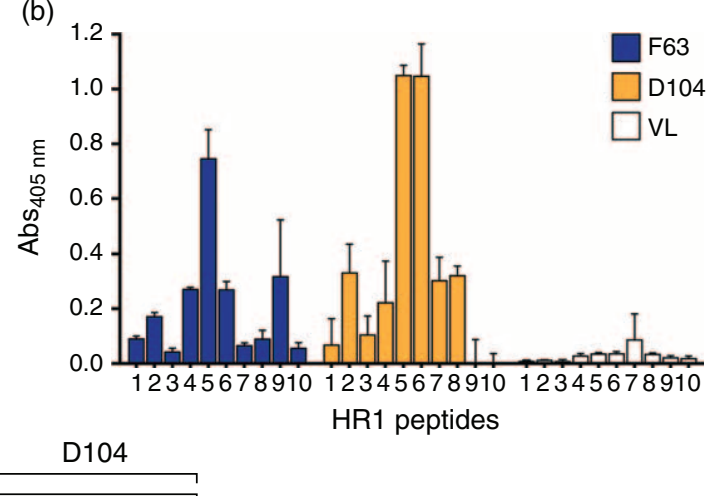

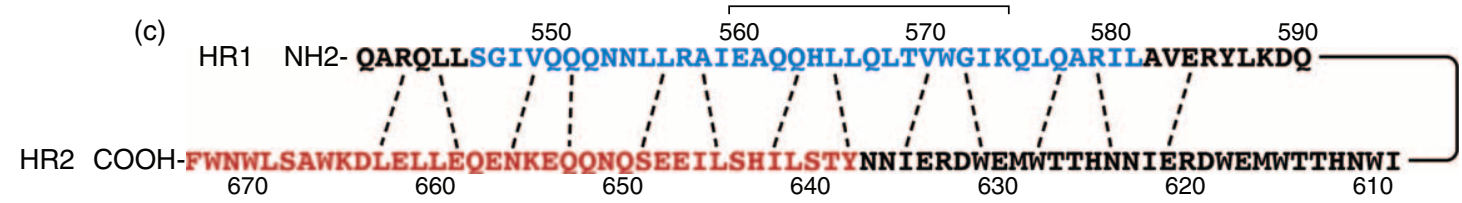

Fig. 2. Epitope mapping of antiviral VLs. (a) Amino acid sequences of the 10 peptides (15-mer) representing the HR1 template (N36 in blue) used as antigens to map the F63 and D104 epitopes. Each peptide comprises 15 residues, 11 amino acids overlapping the subsequent peptide, and an overhang of four amino acids at $\mathrm{N}$-terminal region. (b) Epitope mapping of F63 and D104 by ELISA, using 10 overlapping peptides of HR1 region and BSA as antigens and $V L_{\text {parental }}(V L)$ as negative control. Data are displayed as $A b s$ measurement at $405 \mathrm{~nm}$. To facilitate data representation, HR1 binding was calculated according to the following formula:

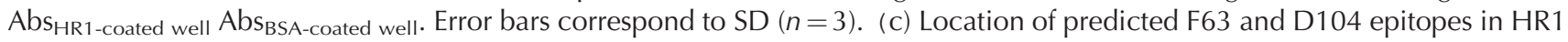
region. Amino acid residues in red constitute the T-20 origin and sequence. Amino acid residues highlighted in blue represent the N36 region. Dash lines represent HR1-HR2 interactions. SD, standard deviation.

We also assessed F63 antiviral activity as a dimer. Surprisingly, F63 dimer did not inhibit HIV-1 $1_{\text {NL4-3 }}$ infection (data not shown), which is probably related to steric restrictions in F63 epitope access.

We then asked whether F63 and D104 could inhibit cell-cell fusion between Env-positive cells (HeLa243env) and adjacent CD4-expressing cells (MAGI) [25]. Similar to $\mathrm{T}-20$ and in contrast to $\mathrm{VL}_{\text {parental, }}$ F63 and D104 impaired HeLa cell-cell fusion in a concentrationdependent manner (Fig. 3c). Fusion between control HeLa273 2 env - without Env expression - and MAGI cells did not occur (data not shown). These results emphasize HIV-1 fusion as the target of F63 and D104.

To test the hypothesis that F63 and D104 were as active as T-20 toward clinically relevant HIV isolates, the $\mathrm{IC}_{50}$ of VLs was evaluated against two HIV-1 and HIV-2 primary isolates in TZM-bl cells. HIV-1 primary isolates belong to distinct subtypes of the major HIV-1 group $\mathrm{M}$, clade J (93AOHDC250) [26] and clade H (93AOCA251) [26]. HIV-2 primary isolates 03PTHCC12 and 10PTHSMNC [26] belong to the most prevalent HIV-2 group [39] (group A, 90\% worldwide). Despite the divergent HR1 sequences of the HIV-1 primary isolates (Fig. S2, http:// links.lww.com/QAD/A912), F63 neutralized both viruses $\left(\mathrm{IC}_{50}\right.$ was $402 \pm 46 \mathrm{nmol} / 1$ for $93 \mathrm{AOHDC} 250$ isolate and $469 \pm 41 \mathrm{nmol} / 1$ for 93AOCA251 isolate; Fig. 4a). Although F63 did not inhibit these HIV-1 primary isolates as potently as $\mathrm{T}-20, \mathrm{IC}_{50}$ values are in the nanomolar range for both $\mathrm{HIV}-1$ subtypes $\left(\mathrm{IC}_{50}\right.$ was
$402 \pm 46 \mathrm{nmol} / 1$ for F63 vs. $1.3 \pm 0.4 \mathrm{nmol} / 1$ for T-20 for 93AOHDC250 isolate; $469 \pm 41 \mathrm{nmol} / 1$ for F63 vs. $0.4 \pm 0.1 \mathrm{nmol} / 1$ for T-20 for 93AOCA251 isolate; Fig. 4a and c). This fact still supports F63 as a potent inhibitor of these two HIV-1 primary isolates. In contrast to T-20, F63 also inhibited the two HIV-2 primary isolates in the nanomolar range $\left(\mathrm{IC}_{50}\right.$ was $460 \pm 19 \mathrm{nmol} /$ 1 for $\mathrm{F} 63$ vs. $2855 \pm 483 \mathrm{nmol} / 1$ for $\mathrm{T}-20$ for 03PTHCC12 isolate; $\mathrm{IC}_{50}$ was $433 \pm 38 \mathrm{nmol} / 1$ for F63 vs. $266 \pm 25 \mathrm{nmol} / 1$ for T-20 for 10PTHSMNC isolate; Fig. 4b and d). In contrast to F63, D104 did not inhibit either HIV-1 or HIV-2 primary isolates (data not shown). These results suggest that in addition to inhibition of HIV-1 non-B subtypes, F63 can be a potent inhibitor of HIV-2 isolates. We also tested the antiviral potency of $\mathrm{F} 63$ in peripheral blood mononuclear cells against a panel of HIV-1 primary isolates from the most prevalent subtypes B (developed countries) and C (developing countries). As shown in Table S3, http:// links.lww.com/QAD/A912, the $\mathrm{IC}_{50}$ values obtained for F63 were similar to T-20 in the nano-picomolar range. These results indicate that F63 also potently inhibits isolates from the most prevalent HIV-1 subtypes in primary lymphocytes, with comparable activity to T-20.

Overall, F63 was as active as T-20 as judged by the $\mathrm{IC}_{50}$ against all tested HIV isolates (no significant $P$ value; Fig. 4e). Moreover, F63 was not significantly less active than T-20 either for HIV-1 or HIV-2 primary isolates (no significant $P$ value; data not shown). These results indicate that F63 presents an antiviral activity similar to T-20. 
(a)

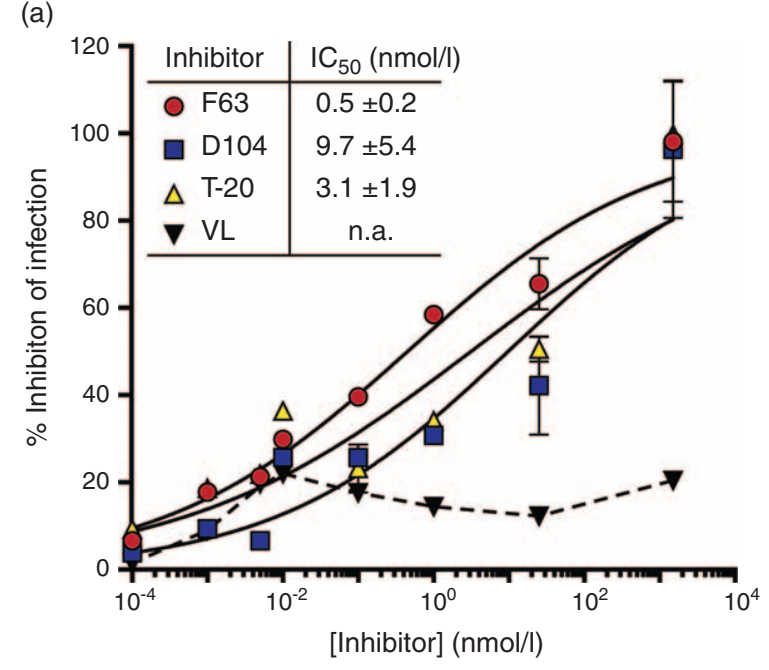

(b)

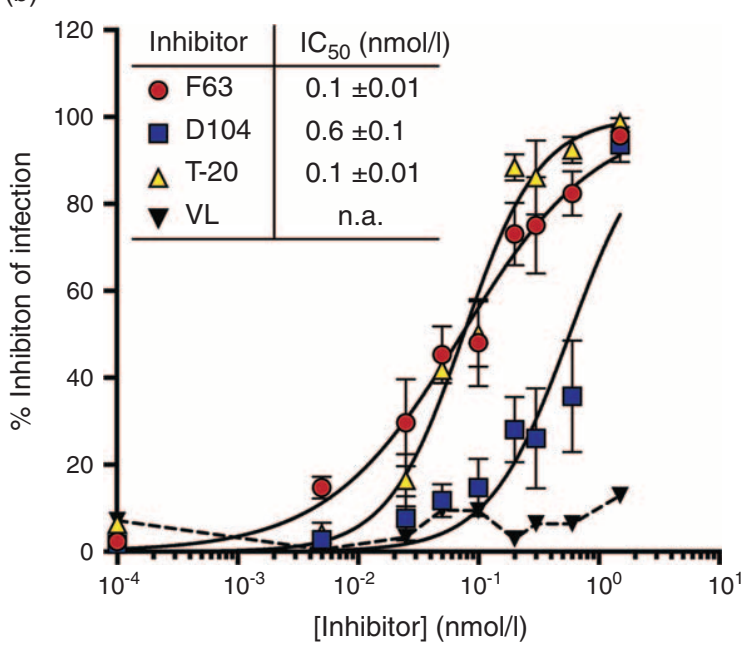

(c)

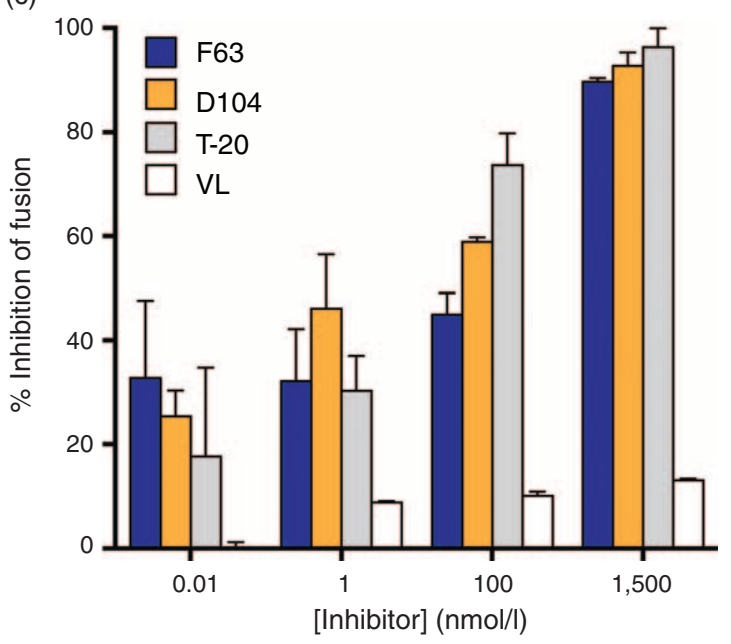

Fig. 3. Antiviral activity of VLs. Percentage of viral infection inhibition was assessed against the laboratory-adapted strain HIV-1 ${ }_{\text {NL4-3 }}$ in TZM-bl (a) and Jurkat cells (b). HIV infectivity was evaluated by $\beta$-galactosidase activity measurement (TZM-bl) or p24 ${ }^{\mathrm{CA}}$ quantification (Jurkat). Data are displayed as percentage of infectivity inhibition (virus/no
To test F63 neutralizing activity against HIV-1 strains resistant to T-20, we evaluated the susceptibility of two $\mathrm{HIV}-1$ variants displaying well defined mutations for T-20 resistance $[38,40]$. HIV-1 variants resistant to T-20 derived from HIV-1 NL4-3 D36G (parental) susceptible to $\mathrm{T}-20[38,40]$. F63 presented no fold increase of $\mathrm{IC}_{50}$ for all tested HIV-1 variants resistant to $\mathrm{T}-20$ relative to parental HIV-1 ( IC $_{50}$ fold increase was 0.66 for NL4-3 (D36G) V38A/N42D; no significant $P$ value and 0.70 for NL4-3 (D36G) V38A/N42T; no significant $P$ value; Fig. S5, http://links.lww.com/QAD/A912). In contrast, $\mathrm{T}-20 \mathrm{IC}_{50}$ was reported to present a fold increase of approximately $3.94 \times 10^{3}$ and $1.61 \times 10^{4}$ for HIV-1 variants NL4-3 (D36G) V38A/N42D and V38A/N42T, respectively, comparing with the parental HIV-1 [6]. These data suggest that F63 could constitute an alternative in the treatment of patients infected with HIV-1 strains resistant to $\mathrm{T}-20$.

\section{F63 interaction with lipid membranes}

As T-20 antiviral mechanism is associated with membrane interaction, we also evaluated the lipid-binding capacity of F63 against membrane model systems mimicking the major lipids of cellular membrane and cholesterol-rich viral envelope [41]. In the presence of lipid membranes, variations in the fluorescent residue emission are typically associated with protein-membrane interactions [42]. Taking advantage of the tryptophan residue (Trp; position 46 in F63 and 37 in $\mathrm{VL}_{\text {parental }}$ Fig. 1e), we performed partition experiments based on the VL quantum yield variations. Fluorescence emission from F63 Trp decreased with increasing lipid concentrations of the both membrane models tested (Fig. $5 \mathrm{a}$ and b). The $K_{\mathrm{p}}$ values retrieved from data fitting with the partition formalism were in the same order of magnitude for viral envelope and cell membrane models (Table S4, http://links.lww. com/QAD/A912). The $K_{\mathrm{p}}$ correlates with the extent of protein interaction with the lipid membrane models ratio between the concentration of a given molecule in two separate and immiscible phases. In contrast, we did not observe variations in the fluorescence emission of $\mathrm{VL}_{\text {parental }} \operatorname{Trp}$ (Fig. 5a and b). These results suggest that

inhibitors $=0 \%$ inhibition; no virus/no inhibitors = backbackground) according to the formula: [1 - $\left(\mathrm{Abs}_{\text {virus/inhibitors }}\right.$ $\left.\left.-\mathrm{Abs}_{\text {background }}\right) /\left(\mathrm{Abs}_{\text {virus/noinhibitors }}-\mathrm{Abs}_{\text {background }}\right)\right] \times 100$. Error bars correspond to SD $(n=3)$. (c) Cell-cell fusion assay. HeLa cells presenting functional gp120/gp41 complexes at cell surface and expression of HIV-1 transactivator of transcription (Tat) protein (HeLa243env) were cocultured with CD4-expressing HeLa cells (MAGI) in the inhibitors presence. Fusion inhibition was assessed by $\beta$-galactosidase activity measurement. Data are displayed as percentage of fusion inhibition (HeLa243env cells $/$ no inhibitors $=0 \%$; no HeLa243env cells/no inhibitors = background) according to the formula: [1-( $\left.\mathrm{Abs}_{\text {HeLa243env/inhibitors }}-\mathrm{Abs}_{\text {background }}\right) /$ $\left.\left(\mathrm{Abs}_{\text {HeLa243env/noinhibitors }}-\mathrm{Abs}_{\text {background }}\right)\right] \times 100$. Error bars correspond to $\mathrm{SD}(n=3)$. SD, standard deviation. 
(a)

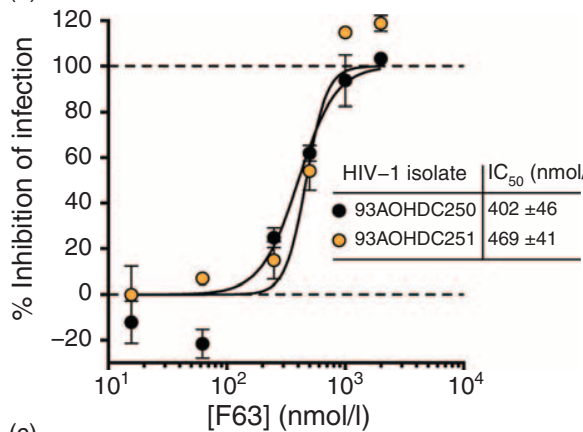

(c)

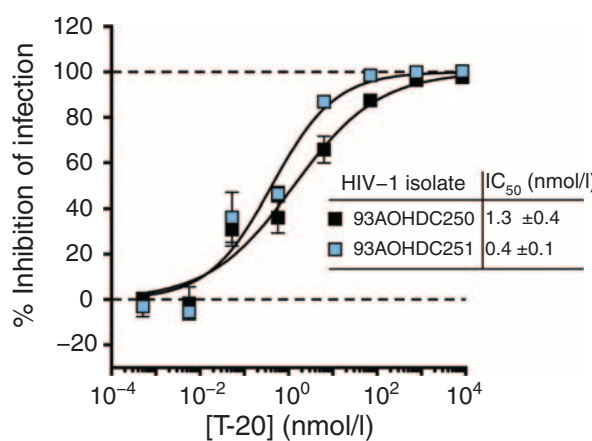

(b)
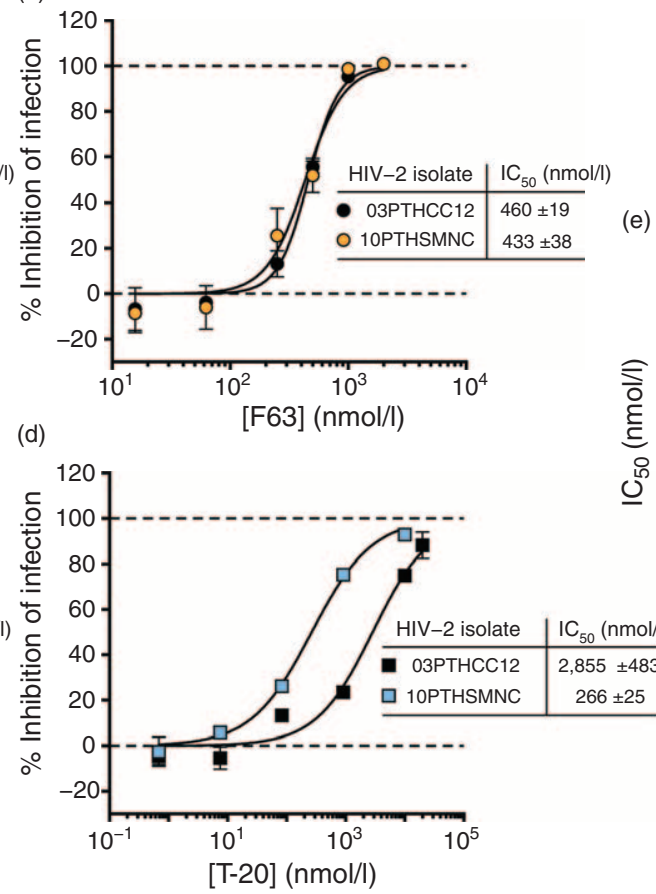

(e)

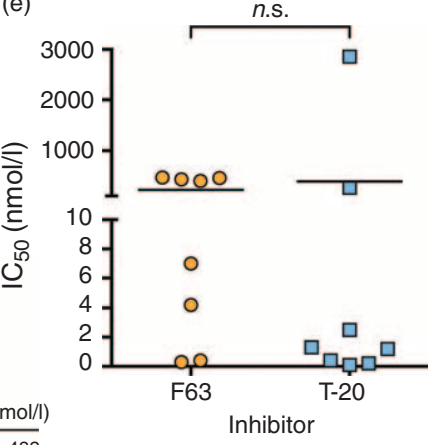

Fig. 4. Antiviral activity of VLs against HIV primary isolates. Antiviral activity of F63 and Food and Drug Administrationapproved T-20 against HIV-1 (a and c) and HIV-2 (b and d) primary isolates. HIV infectivity was evaluated by luciferase activity measurement. Data are displayed as percentage of infectivity inhibition (virus/no inhibitors $=0 \%$; no virus/no inhibitors $=$

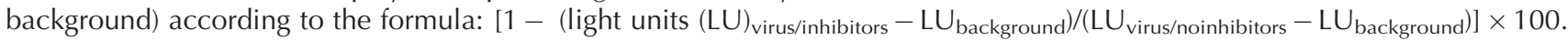
Error bars correspond to $\mathrm{SD}(n=4)$. (e) IC 50 values of $\mathrm{F} 63$ and $\mathrm{T}-20$ for all tested HIV primary isolates $(* P<0.05$; $* * P<0.01$; *** $P<0.001 ; \mathrm{NS}$; $\left.t_{\text {-'test' }}\right)$. Bars represent mean values.

F63 interact with lipid membranes, independently of the cholesterol content. Spectral properties of lipophilic probes such as 4-(2-[6-(dioctylamino)-2-naphthaleny1]ethenyl)-1-(3-sulfopropyl)pyridinium inner salt (di8-ANEPPS), which are responsive to variations in membrane dipole potential, can also be exploited to study protein-membrane interactions [43]. Excitation spectra of di-8-ANEPPS inserted in both membrane models underwent a redshift to higher wavelengths indication of a membrane dipole potential perturbation only in the F63 presence (Fig. $5 \mathrm{c}$ and d). These observations complement the previous partition results and support the hypothesis that F63 has unique membrane-interacting properties, unlike $\mathrm{VL}_{\text {parental }}$. F63 also presented a binding affinity $\left(K_{D}\right)$ of $\sim 8 \mathrm{nmol} / 1$ to $\mathrm{N} 36$ as determined by surface plasmon resonance (Table S5, http://links.lww.com/QAD/A912), establishing this VL as a high-affinity binder in the low nanomolar range.

\section{Discussion}

HIV entry inhibition is a key component of any antiviral therapeutic scheme leading to impairment of de novo infection. In this report, we selected a broad and potent HIV fusion inhibitor from a synthetic repertoire of VL domains. Several studies have shown that VLs tend to aggregate less [12-14] and present higher antigenbinding properties $[44,45]$ than $\mathrm{VH}$ domains. In this study, we went further relative to others $[46,47]$ and successfully selected a high-affinity VL with elongated CDRs. Our data suggest that the screening of libraries containing CDRs-elongated antibody formats result in the selection of cryptic epitope binders, mimicking the longer and more flexible CDRs found in camelids [48].

Here, we took advantage of VLs reduced size to target a sterically restricted region on HR1 of gp41 (N36). Together with the corresponding HR2 region, N36 is sufficient to form the $6 \mathrm{HB}$ structure responsible for the HIV fusion [19]. Accordingly, a VL-N36 interaction would prevent the $6 \mathrm{HB}$ assembly, leading to HIV entry impairment. Epitope mapping of the most potent HIV inhibitors revealed two similar sequences within the N36 central region, previously described as part of a highly conserved cavity (hydrophobic pocket) essential for HR2 binding [19,49]. Despite the location of D104 target region within the F63 target sequence, antiviral activity against HIV primary isolates was only observed with F63. Thus, our data seem to indicate that targeting of D104 epitope is insufficient for broad neutralization of HIV. Nevertheless, as D104 affinity was not determined, we cannot exclude that it may influence viral inhibition. 
(a)

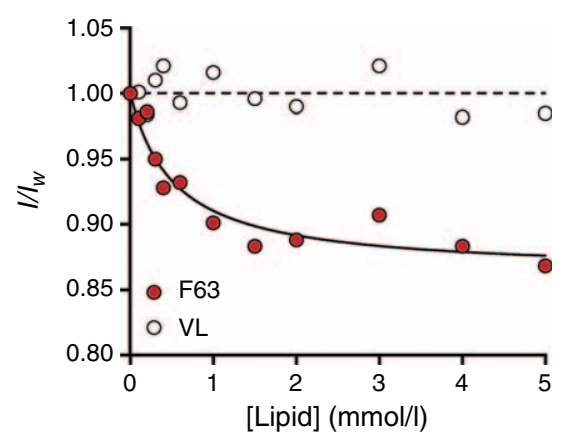

(c)

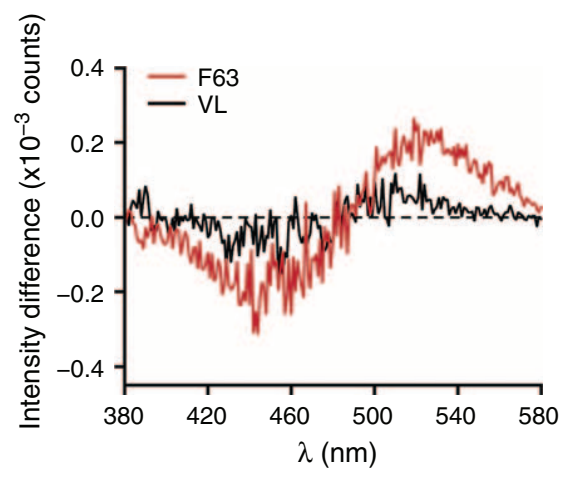

(b)

(d)


Fig. 5. F63 membrane interactions. Partition profiles of $\mathrm{F} 63$ and control $\mathrm{VL}_{\text {parental }}(\mathrm{VL})$ toward $\mathrm{POPC}$ (cellular membrane model) (a) and POPC: cholesterol (2:1; virus envelope model) (b). $\mathrm{F} 63$ and $\mathrm{VL}_{\text {parental }}$ were titrated with small volumes of large unilamellar vesicles (LUV) up to final lipid concentrations, [L]. sdAb intrinsic fluorescence emission, I, was collected for each [L], and normalized to the respective emission in the aqueous media, $I_{\mathrm{W}}$. The line represents the best fit of Eq. (1) (in supplementary material) to one of three independent replicates. Differential excitation spectra of di-8-ANEPPS-labelled POPC (c) and POPC : cholesterol $(2: 1)$ (d) liposomal membrane models in the presence of F63 or control VL $L_{\text {parental }}(\mathrm{VL})$. Graphs were obtained by subtraction of the normalized di-8-ANEPPS excitation spectra controls from the spectra in the presence of each $\mathrm{VL}$ (normalization to the respective spectrum integral). The presented spectra constitute one of three independent replicates. POPC, 1-palmitoyl-2-oleyl-sn-glycero-3-phosphocholine.

On the other hand, F63 epitope represents a promising target with $\sim 60 \%$ conservation amongst HIV-1 subtypes and even HIV types (Fig. S2, http://links.lww.com/ QAD/A912). This predicted epitope is also distinct from the $\mathrm{T}-20$ binding region that has a low genetic barrier to drug resistance, mainly the Gly-Ile-Val sequence $\left(\mathrm{HR} 1_{36-38(\mathrm{HXB} 2)}\right)[38,50]$, as reinforced by the observed F63 inhibition of T-20 resistant HIV-1 strains. Moreover, a substitution of a single residue on $\sim 70 \%$ of the F63 predicted epitope would lead to impaired or nonfunctional HIV-1 entry mutants as reported by Sen et al. [51] (Fig. S2, http://links.lww.com/QAD/A912). F63 epitope conservation and importance for HIV fusion together with the fact that this VL domain inhibited HIV1 primary isolates from distinct subtypes similarly to T-20 and HIV-2 primary isolates highlight F63 potency and predict a high breadth for this inhibitor. Moreover, as T-20 has a limited activity on HIV-2 [6,52], F63 could constitute an alternative to the treatment of this HIV type.

The close proximity of gp41 to viral envelope and cellular membrane during HIV entry questions the role of membranes in gp41-targeting inhibitors mechanism. For example, T-20 shows considerable interaction with lipid membranes [20]. Also, broadly neutralizing antibodies 2F5 and 4E10 are capable of stable epitope binding through cross-reactive lipid interaction [53]. We have assessed F63 membrane interactions through fluorescence spectroscopy methodologies and identified its partition toward lipid membrane models. Interestingly, the $\mathrm{VL}_{\text {parental }}$ was unable to interact with these models, suggesting that this property was acquired during CDRs randomization and is associated to CDR1 and/or CDR3. From a pharmacological standpoint, membranes interaction is a desirable property of an inhibitor mechanism [54], enabling the establishment of local and transient reservoirs both in the viral envelope and cellular membrane. Furthermore, the F63 lack of a Fc immune-triggering domain avoids cross-reactivity with lipids, a significant drawback in 2F5 and 4E10 application [55].

To our knowledge, this is the first report presenting a synthetic VL sdAb designed as a potent inhibitor of HIV infection. Other fusion inhibitors with an antiviral 
activity similar to F63 were already described $[7,56]$. However, F63 combine the reduced molecular weight of small nonantibody inhibitors with the specificity and high-affinity of antibody paratopes and the excellent biophysical properties and versatility of antibody formats. Despite $\mathrm{VHH}$ antibody fragments were also identified as anti-HIV inhibitors [57-61], these variable domains target gp120 and were not synthetically randomized, being derived from llama immunization. Owing to protease resistance and simplicity of sdAbs, F63 may also overcome major T-20 weaknesses, such as oral administration preclusion, high production cost, and short half-life [62,63]. Additionally, F63 potency and biodistribution may be further improved by several strategies such as coupling of effector molecules (enzymes and cytotoxic drugs) and inhibitor targeting to the cholesterol-rich areas where HIV preferentially enters [64], for example, through attachment of cholesterol-binding peptides.

To address the expected immunogenicity of a rabbit VL, we successfully humanized F63 by removing residues potentially recognized as T-cell epitopes (deimmunization) as described in Jones et al. [65]. Humanized F63 neutralized HIV-1 ${ }_{\text {NL4-3 }}$ laboratory-adapted strain similarly to rabbit F63 and proved to be more stable than its rabbit homolog because of alanine substitution of unpaired cysteines performed along with F63 humanization (data not shown). It is conceivable that the rabbitconserved cysteine at position 91 - that forms an unusual disulfide bridge between variable and constant domains [66] - together with the cysteine selected in CDR1 sequence were major contributors to the insolubility of F63 during the purification process. Therefore, our library design strategy could benefit from the replacement of DVN by the NDT degenerate codon, which encodes fewer cysteine residues and does not yield stop codons.

In conclusion, we successfully developed a potent and broad fusion inhibitor of HIV-1 and HIV-2 infection using a VL sdAb as scaffold. We validated the selection of potent inhibitors based on a rational engineering strategy for synthetic library design. Our findings also encourage exploration of CDRs elongation for the design or improvement of next generation HIV inhibitors.

\section{Acknowledgements}

We thank C. Barbas III for kindly providing pComb3x plasmid, O. Schwartz for kindly providing the HeLa243env and HeLa273 2 env cells, and Technophage for kindly providing modified pT7-FLAG-2 and purified $\mathrm{VL}_{\text {parental }}$

C.C-S., T.F., P.B., S.O., C.R., A.C., C.C., Q.S-C., J.AP., C.F., N.T., F.A-S., M.C., A.V., and J.G. conceived and designed the experiments. C.C-S., T.F., P.B., C.R., Q.S-C., and F.A-S. performed the experiments. C.C-S., T.F., P.B., and F.A-S. analyzed the data. C. C-S. and T.F. wrote the article.

This work was supported by grants HIVERA/0002/ 2013, PTDC/SAU-EPI/122400/2010 and VIH/SAU/ 0029/2011 from Fundação para a Ciência e a Tecnologia - Ministério da Educação e Ciência (FCT-MEC), Portugal. C.C-S. and T.F. were supported by FCTMEC PhD fellowships SFRH/BD/73838/2010 and SFRH/52383/2013. F.A-S. and A.S.V. were supported by FCT Investigator Programme IF/01010/2013 and IF/00803/2012.

The following reagents were obtained through the NIH AIDS Reagent Program (Division of AIDS, NIAID, NIH): HIV-1 Subtype B (MN) Env Peptide Set; T-20, Fusion Inhibitor from Roche; pNL4-3 from M. Martin [40]; HIV-1 NL4-3 gp41 D36G Virus from Trimeris, Inc. [38,40]; HIV-1 NL4-3 gp41 (36G) V38A, N42D Virus from Trimeris, Inc. [38,40]; HIV-1 NL4-3 gp41 (36G) V38A, N42T Virus from Trimeris, Inc. [38,40]; TZM-bl from J. C. Kappes, X. Wu and Tranzyme Inc. [5,67-70]; HeLa-CD4-LTR- $\beta$-gal from M. Emerman [71], and Jurkat Clone E6-1 from A. Weiss [72].

Accession codes: The VL F63 sequence reported here has been deposited in the GenBank database (accession number KT119563).

\section{Conflicts of interest}

There are no conflicts of interest.

\section{References}

1. Dando TM, Perry CM. Enfuvirtide. Drugs 2003; 63:2755-2768.

2. Wild C, Oas T, McDanal C, Bolognesi D, Matthews T. A synthetic peptide inhibitor of human immunodeficiency virus replication: correlation between solution structure and viral inhibition. Proc Natl Acad Sci U S A 1992; 89:10537-10541.

3. Matthews TJ, Wild CT, Shugars DC, Greenwell TK, Mcdanal CB. Peptides corresponding to a predictive a-helical domain of human immunodeficiency virus type $1 \mathrm{gp} 4 \mathrm{l}$ are potent inhibitors of virus infection. Proc Natl Acad Sci U S A 1994; 91:97709774.

4. Wilen CB, Tilton JC, Doms RW. HIV: cell binding and entry. Cold Spring Harb Perspect Med 2012; 2:a006866.

5. Wei X, Decker JM, Liu H, Zhang Z, Arani RB, Kilby JM, et al. Emergence of resistant human immunodeficiency virus type 1 in patients receiving fusion inhibitor (T-20) monotherapy. Antimicrob Agents Chemother 2002; 46:1896-1905.

6. Borrego P, Calado R, Marcelino JM, Pereira P, Quintas A, Barroso $\mathrm{H}$, et al. An ancestral HIV-2/simian immunodeficiency virus peptide with potent HIV-1 and HIV-2 fusion inhibitor activity. AIDS 2013; 27:1081-1090.

7. Pang W, Tom SC, Zheng YT. Current peptide HIV type-1 fusion inhibitors. Antivir Chem Chemother 2009; 20:1-18.

8. Ward E, Güssow D, Griffiths A, Jones P, Winter G. Binding activities of a repertoire of single immunoglobulin variable domains secreted from Escherichia coli. Nature 1989; 341:544-546.

9. Kolkman JA, Law DA. Nanobodies: from Ilamas to therapeutic proteins. Drug Discov Today Technol 2010; 7:e95-e146. 
10. de Marco A. Biotechnological applications of recombinant single-domain antibody fragments. Microb Cell Fact 2011; 10:44

11. Kim DY, To R, Kandalaft H, Ding W, Van Faassen H, Luo Y, et al Antibody light chain variable domains and their biophysically improved versions for human immunotherapy. MAbs 2014; 6:219-235.

12. Dubnovitsky AP, Kravchuk ZI, Chumanevich AA, Cozzi A Arosio $\mathrm{P}$, Martsev SP. Expression, refolding, and ferritin-binding activity of the isolated VL-domain of monoclonal antibody F11. Biochemistry (MosC) 2000; 65:1011-1018.

13. Ewert S, Huber T, Honegger A, Plückthun A. Biophysical properties of human antibody variable domains. / $\mathrm{Mol} \mathrm{BiO}$ 2003; 325:531-553.

14. Hussack G, Keklikian A, Alsughayyir J, Hanifi-Moghaddam P, Arbabi-Ghahroudi $M$, van Faassen $\mathrm{H}$, et al. A VL single-domain antibody library shows a high-propensity to yield nonaggregating binders. Protein Eng Des Sel 2012; 25:313-318.

15. Colby DW, Chu Y, Cassady JP, Duennwald M, Zazulak H, Webster JM, et al. Potent inhibition of huntingtin aggregation and cytotoxicity by a disulfide bond-free single-domain intracellular antibody. Proc Natl Acad Sci U S A 2004; 101:1761617621.

16. Schiefner A, Chatwell L, Körner J, Neumaier I, Colby DW Volkmer R, et al. A disulfide-free single-domain VL intrabody with blocking activity towards huntingtin reveals a novel mode of epitope recognition. / Mol Biol 2011; 414:337-355.

17. Lee W-R, Jang J-Y, Kim J-S, Kwon M-H, Kim Y-S. Gene silencing by cell-penetrating, sequence-selective and nucleic-acid hydrolyzing antibodies. Nucleic Acids Res 2009; 38:1596-1609.

18. Paz K, Brennan LA, lacolina M, Doody J, Hadari YR, Zhu Z Human single-domain neutralizing intrabodies directed against Etk kinase: a novel approach to impair cellular transformation. Mol Cancer Ther 2005; 4:1801-1809.

19. Chan DC, Fass D, Berger JM, Kim PS. Core structure of gp41 from the HIV envelope glycoprotein. Cell 1997; 89:263-273.

20. Veiga S, Henriques S, Santos NC, Castanho M. Putative role of membranes in the HIV fusion inhibitor enfuvirtide mode of action at the molecular level. Biochem / 2004: 377:107-110.

21. Martins do Canto AMT, Palace Carvalho AJ, Prates Ramalho JP, Loura LMS. Effect of amphipathic HIV fusion inhibitor peptides on POPC and POPC/cholesterol membrane properties: a molecular simulation study. Int / Mol SCi 2013; 14:14724-14743.

22. Rato S, Maia S, Brito PM, Resende L, Pereira CF, Moita C, et al Novel HIV-1 knockdown targets identified by an enriched kinases/phosphatases shRNA library using a long-term iterative screen in Jurkat T-cells. PLoS One 2010; 5:e9276.

23. Calado M, Matoso P, Santos-Costa Q, Espirito-Santo M, Machado J, Rosado L, et al. Coreceptor usage by HIV-1 and HIV-2 primary isolates: the relevance of CCR8 chemokine receptor as an alternative coreceptor. Virology 2010; 408:174-182.

24. Da Silva FA, Santa-Marta M, Freitas-Vieira A, Mascarenhas P, Barahona I, Moniz-Pereira J, et al. Camelized rabbit-derived VH single-domain intrabodies against Vif strongly neutralize HIV-1 infectivity. I Mol Biol 2004; 340:525-542.

25. Schwartz O, Alizon M, Heard J, Danos O. Impairment of T cell receptor-dependent stimulation in CD4+ lymphocytes after contact with membrane-bound HIV-1 envelope glycoprotein. Virology 1994; 198:360-365.

26. Borrego P, Calado R, Marcelino JM, Bártolo I, Rocha C, CavacoSilva $P$, et al. Baseline susceptibility of primary HIV-2 to entry inhibitors. Antivir Ther 2012; 17:565-570.

27. Hamers-Casterman C, Atarhouch T, Muyldermans S, Robinson G, Hamers C, Songa EB, et al. Naturally occurring antibodies devoid of light chains. Nature 1993; 363:446-448.

28. De Genst E, Silence K, Decanniere K, Conrath K, Loris R, Kinne $\mathrm{J}$, et al. Molecular basis for the preferential cleft recognition by dromedary heavy-chain antibodies. Proc Natl Acad Sci U S A 2006; 103:4586-4591.

29. Gonçalves J, Aires da Silva F. Engineering rabbit antibody variable domains and uses thereof 2008, WO2008136694A1.

30. Goncalves J, Silva F, Freitas-Vieira A, Santa-Marta M, Malhó R, Yang $X$, et al. Functional neutralization of HIV-1 Vif protein by intracellular immunization inhibits reverse transcription and viral replication. / Biol Chem 2002; 277:32036-32045.

31. Kabat E, Wu T, Bilowsky H. Sequences of immunoglobulin chains.. National Institutes for Health: NIH Publication 802008; 1979.
32. Heidmann O, Rougeon F. Immunoglobulin kappa light-chain diversity in rabbit is based on the $3^{\prime}$ length heterogeneity of germ-line variable genes. Nature $1984 ; 311: 74-76$.

33. Momany C, Kovari LC, Prongay AJ, Keller W, Gitti RK, Lee BM, et al. Crystal structure of a camel single-domain $\mathbf{V H}$ antibody fragment in complex with lysozyme. Nature 1996; 3:803-811.

34. Fellouse Fa, Wiesmann C, Sidhu SS. Synthetic antibodies from a four-amino-acid code: a dominant role for tyrosine in antigen recognition. Proc Natl Acad Sci U S A 2004; 101:12467-12472.

35. Birtalan S, Fisher RD, Sidhu SS. The functional capacity of the natural amino acids for molecular recognition. Mol Biosyst 2010; 6:1186-1194.

36. Koide S, Sidhu SS. The importance of being tyrosine: lessons in molecular recognition from minimalist synthetic binding proteins. ACS Chem Biol 2009; 4:325-334.

37. Chan DC, Chutkowski CT, Kim PS. Evidence that a prominent cavity in the coiled coil of HIV type $1 \mathrm{gp} 41$ is an attractive drug target. Proc Natl Acad Sci U S A 1998; 95:15613-15617.

38. Rimsky LT, Shugars DC, Matthews TJ. Determinants of human immunodeficiency virus type 1 resistance to gp41-derived inhibitory peptides. / Virol 1998; 72:986-993.

39. Peeters M, Toure-Kane C, Nkengasong J. Genetic diversity of HIV in Africa: impact on diagnosis, treatment, vaccine development and trials. AIDS 2003; 17:2547-2560.

40. Adachi a, Gendelman HE, Koenig S, Folks T, Willey R, Rabson $A$, et al. Production of acquired immunodeficiency syndromeassociated retrovirus in human and nonhuman cells transfected with an infectious molecular clone. / Virol 1986; 59:284-291.

41. Brügger B, Glass B, Haberkant P, Leibrecht I, Wieland FT, Kräusslich H-G. The HIV lipidome: a raft with an unusual composition. Proc Natl Acad Sci U S A 2006; 103:2641-2646.

42. Figueira TN, Veiga AS, Castanho MARB. The interaction of antibodies with lipid membranes unraveled by fluorescence methodologies. J Mol Struct 2014; 1077:114-120.

43. Matos PM, Franquelim HG, Castanho MARB, Santos NC. Quantitative assessment of peptide-lipid interactions. Ubiquitous fluorescence methodologies. Biochim Biophys Acta 2010; 1798:1999-2012.

44. Brinkmann $U$, Lee B, Pastan I. Recombinant immunotoxins containing the VH or VL domain of monoclonal antibody B3 fused to Pseudomonas exotoxin. J Immunol 1993; 150:27742782.

45. Colby DW, Garg P, Holden T, Chao G, Webster JM, Messer A, et al. Development of a human light chain variable domain (V(L)) intracellular antibody specific for the amino terminus of huntingtin via yeast surface display. J Mol Biol 2004; 342:901912.

46. van den Beucken T, van Neer N, Sablon E, Desmet J, Celis L, Hoogenboom HR, et al. Building novel binding ligands to B7.1 and B7.2 based on human antibody single variable light chain domains. I Mol Biol 2001; 310:591-601.

47. Söderlind E, Vergeles M, Borrebaeck CA. Domain libraries: synthetic diversity for de novo design of antibody V-regions. Gene 1995; 160:269-272.

48. Wesolowski J, Alzogaray V, Reyelt J, Unger M, Juarez K, Urrutia $M$, et al. Single domain antibodies: promising experimental and therapeutic tools in infection and immunity. Med Microbiol Immunol 2009; 198:157-174.

49. Weissenhorn W, Dessen A, Harrison SC, Skehel JJ, Wiley DC. Atomic structure of the ectodomain from HIV-1 gp41. Nature 1997: 387:426-430.

50. Lu J, Deeks SG, Hoh R, Beatty G, Kuritzkes BA, Martin JN, et al. Rapid emergence of enfuvirtide resistance in HIV-1-infected patients: results of a clonal analysis. I Acquir Immune Defic Syndr 2006; 43:60-64.

51. Sen J, Yan T, Wang J, Rong L, Tao L, Caffrey M. Alanine scanning mutagenesis of HIV-1 gp41 heptad repeat 1: insight into the gp120-gp41 interaction. Biochemistry 2010; 49:5057-5065.

52. Witvrouw M, Pannecouque C, Switzer WM, Folks TM, De Clercq E, Heneine W. Susceptibility of HIV-2, SIV and SHIV to various anti-HIV-1 compounds: implications for treatment and postexposure prophylaxis. Antivir Ther 2004; 9:57-65.

53. Franquelim HG, Chiantia S, Veiga AS, Santos NC, Schwille P, Castanho MARB. Anti-HIV-1 antibodies 2F5 and 4E10 interact differently with lipids to bind their epitopes. AIDS 2011; 25:419-428.

54. Vauquelin G, Packeu A. Ligands, their receptors and ... plasma membranes. Mol Cell Endocrinol 2009; 311:1-10. 
55. Verkoczy L, Diaz M. Autoreactivity in HIV-1 broadly neutralizing antibodies: implications for their function and induction by vaccination. Curr Opin HIV AIDS 2014; 9:224-234.

56. McCoy LE, Weiss Ra. Neutralizing antibodies to HIV-1 induced by immunization. / Exp Med 2013; 210:209-223.

57. McCoy LE, Quigley AF, Strokappe NM, Bulmer-Thomas B, Seaman MS, Mortier D, et al. Potent and broad neutralization of HIV-1 by a llama antibody elicited by immunization. J Exp Med 2012; 209:1091-1103.

58. Forsman A, Beirnaert E, Aasa-Chapman MMI, Hoorelbeke B, Hijazi K, Koh W, et al. Llama antibody fragments with crosssubtype human immunodeficiency virus type 1 (HIV-1)-neutralizing properties and high affinity for HIV-1 gp120. J Virol 2008; 82:12069-12081.

59. Hinz A, Hulsik DL, Forsman A, Koh WWL, Belrhali H, Gorlan A, et al. Crystal Structure of the neutralizing Llama VHH D7 and its mode of HIV-1 gp120 interaction. PLOS One 2010 5:28-32.

60. Koh WWL, Steffensen S, Gonzalez-Pajuelo M, Hoorelbeke B, Gorlani A, Szynol A, et al. Generation of a family-specific phage library of llama single chain antibody fragments that neutralize HIV-1. J Biol Chem 2010; 285:19116-19124.

61. Strokappe N, Szynol A, Aasa-Chapman M, Gorlani A, Forsman Quigley A, Hulsik DL, et al. Llama antibody fragments recognizing various epitopes of the $\mathrm{CD} 4 \mathrm{bs}$ neutralize a broad range of HIV-1 subtypes A, B and C. PLoS One 2012; 7:e33298.

62. Kontermann RE. Strategies for extended serum half-life of protein therapeutics. Curr Opin Biotechnol 2011; 22:868-876.

63. Morais M Cantante C, Gano L, Santos I, Lourenco S, Santos C, et al. Biodistribution of a $67 \mathrm{Ga}$-labeled anti-TNF VHH singledomain antibody containing a bacterial albumin-binding domain (Zag). Nucl Med Biol 2014; 41:1-5.

64. Aloia RC, Tiant $\mathrm{H}$, Jensen FC. Lipid composition and fluidity of the human immunodeficiency virus envelope and host cell plasma membranes. Proc Natl Acad Sci U S A 1993; 90:5181-5185.
65. Jones TD, Crompton LJ, Carr FJ, Baker MP. Deimmunization of monoclonal antibodies. In: Clifton N. editor. Methods in molecular biology Cambridge: Humana Press; 2009. pp. 405-423, xiv.

66. McCartney-Francis N, Skurla RM, Mage RG, Bernstein KE. Kappa-chain allotypes and isotypes in the rabbit: cDNA sequences of clones encoding b9 suggest an evolutionary pathway and possible role of the interdomain disulfide bond in quantitative allotype expression. Proc Natl Acad Sci U S A 1984; 81:1794-1798.

67. Platt EJ, Bilska M, Kozak SL, Kabat D, Montefiori DC. Evidence that ecotropic murine leukemia virus contamination in TZM-bl cells does not affect the outcome of neutralizing antibody assays with human immunodeficiency virus type 1. / Virol 2009; 83:8289-8292.

68. Takeuchi Y, McClure MO, Pizzato M. Identification of gammaretroviruses constitutively released from cell lines used for human immunodeficiency virus research. J Virol 2008; 82:12585-12588.

69. Derdeyn CA, Decker JM, Sfakianos JN, Wu X, O'Brien WA, Ratner $L$, et al. Sensitivity of human immunodeficiency virus type 1 to the fusion inhibitor T-20 is modulated by coreceptor specificity defined by the V3 loop of gp120. / Virol 2000; 74:8358-8367.

70. Platt EJ, Wehrly K, Kuhmann SE, Chesebro B, Kabat D. Effects of CCR5 and CD4 cell surface concentrations on infections by macrophagetropic isolates of human immunodeficiency virus type 1. J Virol 1998; 72:2855-2864.

71. Kimpton J, Emerman M. Detection of replication-competent and pseudotyped human immunodeficiency virus with a sensitive cell line on the basis of activation of an integrated betagalactosidase gene. I Virol 1992; 66:2232-2239.

72. Weiss A, Wiskocil RL, Stobo JD. The role of T3 surface molecules in the activation of human T cells: a two-stimulus requirement for IL 2 production reflects events occurring at a pretranslational level. J Immunol 1984; 133:123-128. 